\title{
Left atrial posterior wall isolation: the icing on the cake
}

\author{
Rong Bai ${ }^{1}$
}

Received: 6 April 2016 / Accepted: 20 April 2016/Published online: 16 May 2016

(C) Springer Science+Business Media New York 2016

Catheter ablation for symptomatic, drug-resistant atrial fibrillation (AF) has emerged as a promising new therapeutic option over the past decade. Most paroxysmal AF (PAF) is initiated by premature beats from the pulmonary veins (PVs). Therefore, catheter ablation that electrically isolates PVs could abolish the arrhythmia. However, especially in more server cases like persistent or long-standing persistent AF (Per AF), $\mathrm{PVs}$ are not the only source and non-PV triggers may arise from any part of the heart but most commonly from the coronary sinus, left atrial posterior wall (LAPW), left atrial appendage (LAA), and superior vena cava (SVC) [1]. These structures also serve as substrates to maintain AF episodes after they start. In view of this, it is recommended by the consensus statements that substrate modification should be considered on top of PVs isolation (PVI) in Per AF cases [2]. There is no unique technique of atrial substrate modification while LAPW isolation has been adapted at many centers worldwide and shown to improve the success rate of AF ablation by many isolated studies [3-5]. In this issue of JICE, He et al. reported their results of a meta-analysis on the efficacy and safety profile of adding LAPW isolation to PVI during AF ablation procedures [6]. Five studies with 594 AF patients were included, and the pooled data clearly showed that LAPW isolation reduces atrial tachyarrhythmia recurrence with comparable procedure-related complications and procedural time associated with PVI-alone strategy. Although sample size is small compared to other AF-related trials, this study included the largest series of AF patients treated with LAPW

Rong Bai

bairong74@gmail.com

1 Department of Cardiology, Beijing Anzhen Hospital, Capital Medical University, Beijing 100029, People's Republic of China isolation + PVI strategy and provided solid evidence favoring this approach. Before data from a large-scale randomized controlled clinical trial become available, He et al.'s paper can potentially represent a reference for this topic.

\section{Rationale of performing LAPW isolation}

While we are emphasizing that PVs play a crucial role in the development of AF, we should remember that the LAPW and the PVs are embryological "siblings." Actually, each PV we see in adult forms by two parts that join together during the human heart development. A primitive vein sprouts out of the LA, which bifurcates twice to give four PVs which grow towards the developing lungs. A plexus of veins is formed in the mesoderm enveloping the bronchial buds; these veins will meet with the developing PVs out of the left atrium to establish a connection at the fifth week of gestation. As the left atrium (LA) develops, it progressively incorporates the common PV into the LA wall until all four PVs enter the LAPW separately. The incorporated PVs form the smooth posterior wall of the LA, while the trabeculated portion of the LA comes to occupy a more ventral aspect $[7,8]$. Anatomically, there is an abrupt change in LA subendocardial fiber orientation as this bundle traverses the posterior LA between the PVs, which create a basis of reentry $[9,10]$. Indeed, previous work using noncontact mapping has demonstrated significant conduction abnormalities in the posterior LA during sinus rhythm in patients with PAF [9]. Additionally, the LAPW myocytes have a higher incidence of delayed afterdepolarizations, larger late sodium currents, but smaller inward rectifier potassium currents. The LAPW myocytes also have larger intracellular $\mathrm{Ca}^{2+}$ transient and sarcoplasmic reticulum $\mathrm{Ca}^{2+}$ contents but a less protein expression of $\mathrm{Na}-\mathrm{Ca}$ exchanger [11]. It is not uncommon to see firings from the LAPW initiating AF 
episodes. Hence, underlying tissue architecture and electrophysiological characters in the LAPW may form the substrate for onset and maintenance of AF. When AF becomes sustained, atrial remodeling including fibrosis, fatty and lymphomononuclear infiltration are more pronounced in the LA septum and LAPW $[12,13]$. Acknowledging the aforementioned, we are confident that the LAPW should be considered as a target following PVI when ablating AF. It is expected that this approach will result in a more favorable outcome as compared to PVI-alone. The question being left unanswered, even by He et al.'s study, is whether we should include LAPW isolation in all AF ablation procedures as data has shown PVI-alone is sufficient to eliminate PAF. It seems reasonable to add LAPW isolation on top of PVI if AF has progressed to an advanced stage and the LA has undergone significant remodeling, as in the cases we recently described [5].

\section{Technique to achieve LAPW isolation}

To modify AF substrate in the LAPW, electrically isolating this structure is the goal. Issues remaining controversial include how to perform LAPW ablation and what is the definition of LAPW isolation. The so-called box ablation is derived from the Cox maze IV surgical procedure [14]. It connects bilateral PV-encircling lesions by placing two linear lesion sets both superiorly on the roof and inferiorly at the bottom of the LA. In this way, the entire LAPW is believed to be isolated from the rest of the atria which can be confirmed by an entrance block of the LAPW with or without exit block. The technique developed by Natale's group, however, requires more extensive ablation targeting all near-field potentials in the LAPW. Both entrance block and electrical silence of the LAPW are used as the endpoint of Natale's approach [15]. The study by Dr. He et al. failed to distinguish these techniques from each other due to small sample size and might introduce bias. Because of the complex architecture of the atrial musculature, electrical isolation of the LAPW by a set of linear lesions is always technically difficult. Gaps are unavoidable on ablation lines and dormant conduction may become manifested in the following months. Posterior interatrial connections also allow firings in the LAPW to penetrate epicardially and trigger AF episodes [9]. Therefore, elimination of all LAPW electrical activations is more likely associated with long-term benefits.

\section{Safety of LAPW ablation}

He et al.'s study indicated that procedural time and rate of procedure-related complications did not differ between PVIalone and PVI + LAPW isolation strategies, regardless of the technique utilized for LAPW isolation. One may question on the LA mechanical function after LAPW isolation. However, it is important to understand that the contractility of the LA mainly rely on the anterior portion of LA wall while the LAPW has limited contribution. Another unique, rare but lethal complication associated with extensive LAPW ablation is atrial-esophageal fistula. Caution should be taken when pursuing LAPW isolation and new technologies including contact force and esophageal temperature monitoring will help minimize this complication.

Given the embryological homology of the PVs and the LAPW, and the primary electrophysiological role of these two structures in the development of $\mathrm{AF}$, it is reasonable to include LAPW isolation in AF ablation procedures in addition to PVI, at least in cases with non-paroxysmal AF. Further studies, especially randomized controlled clinical trials, are warranted to identify the optimal strategy to achieve LAPW isolation.

\section{Compliance with ethical standards}

Funding This work was supported the "National Natural Science Foundation of China" (NSFC-81370290), the "Beijing Natural Science Foundation" (7161003), and the "Capital Health Research and Development of Special (2016-2-2062)." Dr. Bai is supported by the Program of Beijing High-Caliber Talent from Overseas (BHTO201410007) and is an awardee of the Overseas High-Level Talent of the Phoenix Plan of the Chaoyang District, Beijing.

\section{References}

1. Zhao Y, Di Biase L, Trivedi C, Mohanty S, Bai R, Mohanty P, et al. Importance of non-pulmonary vein triggers ablation to achieve long-term freedom from paroxysmal atrial fibrillation in patients with low ejection fraction. Heart Rhythm. 2016;13(1):141-9.

2. January CT, Wann LS, Alpert JS, Calkins H, Cigarroa JE, Cleveland Jr JC, et al. 2014 AHA/ACC/HRS guideline for the management of patients with atrial fibrillation: a report of the American College of Cardiology/American Heart Association Task Force on Practice Guidelines and the Heart Rhythm Society. J Am Coll Cardiol. 2014;64(21):e1-76.

3. Kim JS, Shin SY, Na JO, Choi CU, Kim SH, et al. Does isolation of the left atrial posterior wall improve clinical outcomes after radiofrequency catheter ablation for persistent atrial fibrillation?: a prospective randomized clinical trial. Int J Cardiol. 2015;181:277-83.

4. Lim TW, Koay CH, See VA, McCall R, Chik W, Zecchin R, et al. Single-ring posterior left atrial (box) isolation results in a different mode of recurrence compared with wide antral pulmonary vein isolation on long-term follow-up longer atrial fibrillation-free survival time but similar survival time free of any atrial arrhythmia. Circ Arrhythm Electrophysiol. 2012;5(5):968-77.

5. Bai R, Di Biase L, Mohanty P, Trivedi C, Dello Russo A, Themistoclakis S, et al. Proven isolation of the pulmonary vein antrum with or without left atrial posterior wall isolation in patients with persistent atrial fibrillation. Heart Rhythm. 2016;13(1):13240. 
6. He X, Zhou Y, Chen Y, Wu L, Huang Y, He J. (2016). Left atrial posterior wall isolation reduces the recurrence of atrial fibrillation: a meta-analysis. J Interv Card Electrophysiol, 2016 Mar 12. [Epub ahead of print]

7. Colvin EV. (1998). Cardiac embryology. In: Garson AJr (Eds.), The science and practice of pediatric cardiology, 2nd ed. (pp 91-126). Baltimore, Williams \& Wilkins.

8. Pensky B. Review of medical embryology (pp 291-355). New York: McMillan; 1982.

9. Markides V, Schilling RJ, Ho SY, Chow AW, Davies DW, Peters NS. Characterization of left atrial activation in the intact human heart. Circulation. 2003;107(5):733-9.

10. Todd DM, Skanes AC, Guiraudon G, Guiraudon C, Krahn AD, Yee $\mathrm{R}$, et al. Role of the posterior left atrium and pulmonary veins in human lone atrial fibrillation: electrophysiological and pathological data from patients undergoing atrial fibrillation surgery. Circ. 2003;108(25):3108-14.
11. Suenari K, Chen YC, Kao YH, Cheng CC, Lin YK, Chen YJ, et al. Discrepant electrophysiological characteristics and calcium homeostasis of left atrial anterior and posterior myocytes. Basic Res Cardiol. 2011;106(1):65-74.

12. Rohr S. Arrhythmogenic implications of fibroblast-myocyte interactions. Circ Arrhythm Electrophysiol. 2012;5(2):44252.

13. Wilber DJ. Fibroblasts, focal triggers, and persistent atrial fibrillation: is there a connection? Circ Arrhythm Electrophysiol. 2012;5(2):249-51.

14. Reddy VY, Neuzil P, D'Avila A, Ruskin JN. Isolating the posterior left atrium and pulmonary veins with a "box" lesion set: use of epicardial ablation to complete electrical isolation. J Cardiovasc Electrophysiol. 2008;19(3):326-9.

15. Di Biase L, Santangeli P, Natale A. How to ablate longstanding persistent atrial fibrillation? Curr Opin Cardiol. 2013;28(1):26-35. 